$12,997(78 \%)$ and $13,145(71 \%)$ had a cohort participating partner, respectively. However, partner participation was much lower in BiB: 3131/11,538 pregnancies (27\%) had a cohort participating partner. Consistently across all cohorts, in pregnancies with cohort participating partners, mothers were more likely to be living with their partner before birth of the child, be white and have a university degree. They were consistently less likely to have no qualifications and their babies were less likely to be born preterm or have a low birthweight. We saw relatively stable effect estimates within cohorts for associations between maternal smoking and offspring birthweight regardless of whether we used the full sample, the sample where fathers participated (selected either through stratification or adjusting for partner smoking), or the sample where fathers did not participate.

Conclusion Overall, these results show partner selection is unlikely to cause strong selection bias in estimates of maternal effects. This suggests that, although inclusion of partner data with high levels of non-random missingness has the potential to introduce selection bias, in practice, the biasing effect appears to be small. This also has implications for studies of partner effects in their own right.

\section{P64 HOW DO CHANGES IN INDIVIDUAL OR HOUSEHOLD INCOME IMPACT ON MENTAL HEALTH FOR WORKING- AGE ADULTS? A SYSTEMATIC REVIEW}

Rachel M Thomson*, Erik Igelström, Amrit Kaur Purba, Michal Shimonovich, Anna Pearce, Alastair Leyland, Hilary Thomson, S Vittal Katikireddi. MRC/CSO Social and Public Health Sciences Unit, University of Glasgow, Glasgow, UK

\subsection{6/jech-2021-SSMabstracts. 152}

Background Lower incomes are associated with poorer mental health $(\mathrm{MH})$ and wellbeing, but the extent to which income has a causal effect (rather than reflecting reverse causation or confounding factors such as education) is debated. We synthesised evidence from studies measuring the impact of changes in individual or household incomes on $\mathrm{MH}$ and wellbeing for working-age adults (16-64 years).

Methods We searched MEDLINE, Embase, Web of Science, PsycINFO, ASSIA, EconLit and RePEc for randomised controlled trials (RCTs) and quantitative non-randomised studies (NRSs) - PROSPERO registration CRD42020168379. We included studies measuring effects of income change on any $\mathrm{MH}$ or wellbeing outcome. Screening and risk of bias (RoB) assessment were completed independently by two reviewers, using ROBINS-I for NRSs and RoB-2 for RCTs. As per Cochrane guidance, we conducted narrative synthesis based on direction of effects (benefit vs harm) for relevant datapoint(s) within each included study, and compared findings by RoB. Meta-analysis is in progress.

Results Of 16,521 hits screened, 17 RCTs and 118 NRSs (67.0\% longitudinal) were included. Most studies were from high-income settings (71.9\%), with $26.7 \%$ from USA. RoB was high: 102 studies (75.6\%) were rated serious/critical, with confounding being the highest-rated RoB domain in $81.1 \%$ of these studies. Where known $(53.7 \%$ of studies), the most common income sources studied were cash transfers (20.2\%), natural disasters/welfare policy changes $(7.5 \%)$ and lottery wins (5.2\%). For mental health, $80.4 \%$ of 112 datapoints reported beneficial effects of income (95\% CI 73.0-87.7\%, sign test $\mathrm{p}<0.001)$. For wellbeing outcomes $86.5 \%$ of 74 datapoints reported a beneficial effect (95\%CI 78.7-94.3\%, $\mathrm{p}<0.001)$. However, effect sizes were small: for low/moderate RoB datapoints, binary exposures (i.e. increases/decreases in income of any amount) were associated with median 0.089 standard deviation (SD) improvement/worsening in outcome respectively (IQR 0.162, $\mathrm{n}=24$ ); a $1 \mathrm{SD}$ increase/decrease in continuous exposures was associated with median $0.008 \mathrm{SD}$ improvement/worsening (IQR 0.276, $n=4$ ). Studies with low/ moderate RoB were slightly less likely to report beneficial effects compared with serious/critical RoB studies $(77.6 \%$ vs $84.7 \%, \mathrm{p}=0.257)$.

Conclusion Income increases are linked to improved $\mathrm{MH}$ and wellbeing, but on preliminary synthesis effect sizes appear small in the most robust studies. Income provision alone may not be adequate to improve mental health - given that more generous welfare policies are known to be linked with better population $\mathrm{MH}$, it is possible other elements such as conditionality or financial security are also important.

\section{P65 POTENTIAL ALCOHOL USE DISORDER (AUD) AMONG MEN WHO HAVE SEX WITH MEN (MSM) IN IRELAND - FINDINGS FROM THE EUROPEAN MSM INTERNET SURVEY (EMIS) 2017}

\begin{abstract}
${ }^{1,2}{ }^{2}$ Fionn P Daly* ${ }^{3}$ Kate O'Donnell, ${ }^{2,4}$ Martin P Davoren, ${ }^{5}$ Chris Noone, ${ }^{6}$ Peter Weatherburn, ${ }^{7}$ Mick Quinlan, ${ }^{7}$ Bill Foley, ${ }^{3}$ Derval Igoe, ${ }^{2,8}$ Peter M Barrett. ${ }^{1}$ School of Medicine and Health, University College Cork, Cork, Ireland; ${ }^{2}$ School of Public Health, University College Cork, Cork, Ireland; ${ }^{3}$ Health Protection Surveillance Centre, Health Service Executive, Dublin, Ireland; ${ }^{4}$ Sexual Health Centre, Ireland, Cork, Ireland; ${ }^{5}$ School of Psychology, National University of Ireland Galway, Galway, Ireland; ${ }^{6}$ Sigma Research, London School of Hygiene and Tropical Medicine, London, UK; ${ }^{7}$ Gay Health Network, Ireland, Dublin, Ireland; ${ }^{8}$ Department of Public Health HSE-South, St. Finbarr's Hospital, Cork, Ireland
\end{abstract}

\subsection{6/jech-2021-SSMabstracts. 153}

Background Alcohol consumption is a major public health concern in Ireland. Alcohol use disorder (AUD) disproportionately affects men who have sex with men (MSM). However, little is known about the prevalence of AUD in this group in Ireland specifically, and the characteristics of MSM who may struggle with this.

Methods The European MSM Internet Survey 2017 was an online, self-completed, anonymous questionnaire among MSM in Ireland. Standardised questions were used to explore a variety of topics. The validated CAGE-4 questionnaire was used to screen for potential AUD, defined as a CAGE-4 score of $\geq 2$ out of 4 . Multivariable-adjusted logistic regression analysis was used to identify factors associated with potential AUD.

Results In total, 1,793 MSM met inclusion criteria, and $31 \%$ screened positive for AUD. We observed higher odds of possible AUD among MSM who were bisexual (vs. gay/ homosexual) (aOR $1.4895 \% \mathrm{CI} 1.01-2.18$ ), native to Ireland (vs. non-native) (aOR 1.49 95\% CI 1.12-1.96), unemployed (vs. employed) (aOR 1.80 95\%CI 1.02-3.16), had used illicit drugs in the previous year (vs. none) (cannabis only, aOR 1.74 95\%CI 1.14-2.63) (other illicit drugs, aOR 2.28 95\%CI 1.67-3.09), reported anxiety/depression (vs. none) (aOR 1.73 95\%CI 1.12-2.66), and MSM who experienced homophobic abuse (vs. never) (aOR 1.55 95\%CI 1.09-2.22). Student MSM were less likely to screen positive for AUD (vs. employed) (aOR 0.65 95\%CI $0.46-$ $0.93)$.

Conclusion The prevalence of AUD appears to be higher in the MSM population compared to the general male 
population in Ireland. Targeted interventions, such as preventive messaging in collaboration with key LGBT + community and health service partners, may be warranted to reduce the burden of AUD among MSM.

\section{P66 TRANSLATION AND VALIDATION OF THE SWAHILI WARWICK EDINBURGH MENTAL WELLBEING SCALE (WEMWBS) AND DISTRIBUTION OF MENTAL WELLBEING IN ADOLESCENTS AND ADULTS TAKING PART IN THE GIRLS' EDUCATION CHALLENGE PROJECT IN TANZANIA}

\begin{abstract}
${ }^{1}$ Louise Banham, ${ }^{2}$ Elizabeth Bruno-McClung, ${ }^{3}$ Deus Kapinga, ${ }^{2}$ Oyinlola Oyebode*, ${ }^{2}$ Sarah Stewart-Brown, ${ }^{4}$ Manuel Torres-Sahli, ${ }^{3}$ Lydia Wilbard, ${ }^{2}$ Rebecca Willans. ${ }^{1}$ UK Foreign, Commonwealth and Development Office, Dar Es Salaam, Tanzania; ${ }^{2}$ Warwick Medical School, University of Warwick, Coventry, UK; ${ }^{3}$ CAMFED Campaign for female education, Dar Es Salaam, Tanzania; ${ }^{4}$ School of Social Sciences and Humanities, University of Loughborough, Loughborough, UK
\end{abstract}

\subsection{6/jech-2021-SSMabstracts. 154}

Background The Warwick Edinburgh Mental Wellbeing Scale (WEMWBS) is validated for measuring mental wellbeing in UK populations aged $11+$ and has been translated into $30+$ languages. The primary aim of this study was to translate and validate WEMWBS for use in Swahili-speaking populations. This will facilitate measurement and understanding of wellbeing in these populations, evaluation of policy and practice, and enable international comparisons. The secondary aim was to describe mental wellbeing in the participants of the Girls' Education Challenge (GEC) project in Tanzania, run by CAMFED and funded through the UK's Foreign, Commonwealth and Development Office. Specifically, to examine sociodemographic characteristics associated with higher and lower wellbeing in this population.

Methods We created a short questionnaire including WEMWBS and similar scales for comparison, socio-demographic information and self-reported health. This was translated into Swahili using gold standard methodology. We aimed to use this tool to collect data from secondary school students (girls and boys), learner guides, teacher mentors and teachers taking part in the GEC programme in Tanzania. Quantitative data analysis examined internal consistency of WEMWBS, correlation with comparator scales and confirmatory factor analysis. Qualitative work to assess acceptability and comprehensibility of WEMWBS and conceptual understanding of mental wellbeing was carried out through focus groups with GEC participants. These were audio-taped, transcribed and analysed thematically. Finally, we used multivariable logistic regression to explore associations between individual characteristics and 'low' and 'high' mental wellbeing, defined as the lowest and highest quartile of WEMWBS scores.

Results 3052 students were recruited into the study and 574 adults. Both WEMWBS and its short form met quantitative test of reliability and validity. They were correlated with comparator scales and met the criteria to determine that they were measuring one factor. Overall, WEMWBS seemed applicable, understood and relevant to the focus groups of students, learner guides and teachers. For students in the Girls Education Challenge supported government schools: being male, being urban, the absence of markers of marginality and better self-reported health were all significantly associated with better mental wellbeing. Mental wellbeing is higher in students in the final two 'forms' of school compared with the first two. For adults: being urban and better self-reported health were associated with better mental wellbeing.

Conclusion The Swahili translation of WEMWBS is available for use in Swahili speaking populations. Further work to explore how to intervene to increase mental wellbeing in vulnerable GEC participants is needed.

\section{\begin{tabular}{l|l} 
P67 & CLASS-BASED NEIGHBOURHOOD MINORITY STATUS
\end{tabular} PREDICTS MENTAL HEALTH, FOR CULTURAL BUT NOT ECONOMIC COMPONENTS OF CLASS}

${ }^{1}$ Christopher WN Saville*, ${ }^{2}$ Robin Mann. 'North Wales Clinical Psychology Programme, School of Psychology, Bangor University, Bangor, UK; ${ }^{2}$ School of History, Philosophy, and Social Sciences, Bangor University, Bangor, UK

\subsection{6/jech-2021-SSMabstracts. 155}

Background A considerable literature of ethnic density studies links mental health to ethnic minority status in one's neighbourhood. This design has also been applied to other types of identity, such as sexual minorities and Brexit identities, but surprisingly little attention has been paid to class-based identities. Bourdieu decomposes class into several distinct, but mutually reinforcing, forms of capital. Here, we examine whether a 'class density' association with mental health can be found for economic capital - people's material assets and cultural capital - symbolic signifiers of class such as tastes and interests.

Methods Multi-level regression with post-stratification was used to make area-level estimates of cultural and economic capital for each middle super output area in Wales using data from the National Survey for Wales 2017/18 ( $N=11381)$. Mixed effects models were fitted to economic capital, operationalised using respondents' income, house ownership, and material deprivation, and cultural capital, operationalised using items on attendance at a variety of artistic, cultural, and heritage activities. Terms for demographic and area-level variables, as well as random effects of middle super output area were estimated and post-stratified using census to create estimates of area-level economic and cultural capital for all Welsh middle super output areas. These estimates were linked to independent individual-level data from the 2018/19 edition of the survey $(\mathrm{N}=4058)$. Mixed effects models containing individuallevel capital, area-level capital, and their interaction were fitted, predicting whether respondents reported a mental health problem. Models were fitted unadjusted, adjusted for age and gender, adjusted for the other form of capital on the individual and area levels, and adjusted for all these and the other capital's cross-level interaction. Multiple imputation was used to account for missing data.

Results For cultural capital, a cross-level interaction was found where area-level cultural capital was protective in respondents reporting higher levels of individual-level cultural capital, but a risk factor for mental health problems in those with low individual-level cultural capital (odds ratio $=.83, \mathrm{CI}_{95 \%}=.74$ .93). No such relationship was found for economic capital (odds ratio $=.96, \mathrm{CI}_{95 \%}=.88-1.06$ ). These results remained robust in the adjusted models.

Conclusion The presence of a class density association with mental health for cultural capital but not economic capital 